\title{
A Fourier Analysis of the Interacting Pair of Galaxies KPG 404 (NGC 5394/95)
}

\author{
Ivânio Puerari \\ INAOE, Calle Luis Enrique Erro \#1, 72840 Tonantzintla, Pue, Mexico \\ Margarita Valdez-Gutiérrez \\ Departamento de Astronomía, Universidad de Guanajuato, Apartado \\ Postal 144, 36000 Guanajuato, Gto, Mexico
}

Izbeth Hernández-López

INAOE, Calle Luis Enrique Erro \#1, 72840 Tonantzintla, Pue, Mexico

\begin{abstract}
We present a bi-dimensional Fourier analysis of near-infrared images of KPG 404 (NGC 5394/95). The 2D Fourier analysis shows that NGC 5394 is a $\mathrm{H} 2 \beta$ galaxy in the DP classification (Block \& Puerari 1999). In contrast, NGC 5395 displays a very complex structure which needs a number of Fourier coefficients to be explained. A tightly wound $m=1$ (DP class $\mathrm{H} 1 \alpha$ ) is the main structure, but other $m=1$ and $m=2$ coefficients (suggesting spiral arm modulation) are also present in the Fourier spectra. The complex structure of NGC 5395 also suggests a strong interaction in the pair. The $m=1$ coefficients can represent a pseudo ring-type structure, resulting of a collision rather than a grazing passage. We conclude that the most probable scenario of the interaction in this pair should take into account a crossing of NGC 5394 through the disk of NGC 5395.
\end{abstract}

Near infrared studies of galaxies unveil the stellar mass distribution and morphology, hidden at optical wavelengths by high dust extinction (absorption + scattering). In particular, near infrared observations of interacting galaxies contribute to the understanding of what effects interactions have on galactic structure and the morphological evolution of the old stellar component (Block et al. 1994, Block \& Puerari 1999, Hernández-Toledo \& Puerari 2001). In the present work we report our results on the galaxy pair KPG 404 (NGC 5394/95) (Karachentsev 1972). The images were taken using the near-infrared camera CAMILA (Cruz-González et al. 1994) at the $2.1 \mathrm{~m}$ telescope of the Observatorio Astronómico Nacional at San Pedro Mártir, Baja California, México. This is part of a wider project which aims to study near infrared morphological properties in northern interacting galaxy pairs.

In Figure 1 we show the Fourier spectra for $\mathrm{K}^{\prime}$ for both galaxies in the pair. NGC 5394 shows a classical $m=2$ spiral structure, but NGC 5395 displays a complex spectrum: the main peak is for $m=1$, showing also a peak at opposite $p$ suggesting spiral arm modulation (Puerari et al. 2000). The spectrum for $m=2$ shows a similar behaviour. Spectra for the other filters are similar and are not 
shown here. The complex structure of NGC 5395 is possibly a result of a strong interaction between the galaxies. The most plausible scenario is a collision in which NGC 5394 crossed the disk of NGC 5395 (a Cartwheel-type interaction). KPG 404 was included in a sample of M51-type pairs by Laurikainen \& Salo (2000). Nevertheless, loose encounters, passages or M51-type interactions are not the probable responsible of structures as those we see in the evolved old stellar population II of NGC 5395 .
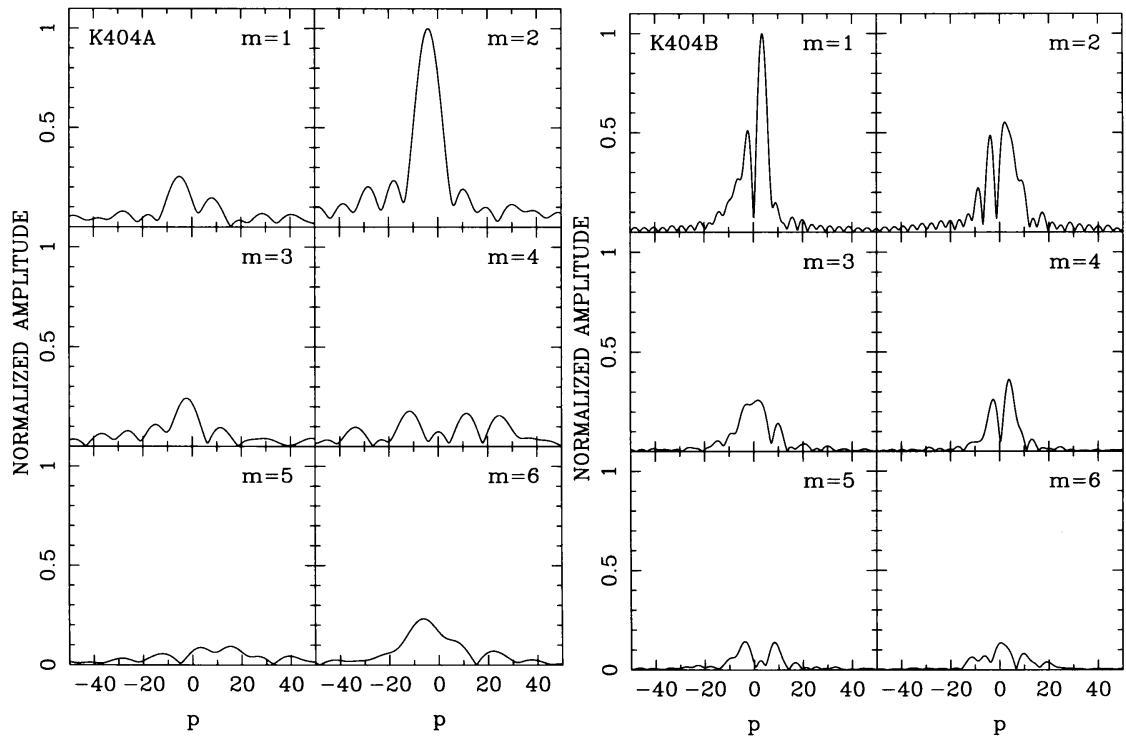

Figure 1. Fourier spectra for $\mathrm{K}^{\prime}$ for both galaxies in the pair. Note the classical $m=2$ spiral for KPG 404A (NGC 5394) and a much more complex spectrum for KPG 404B (NGC 5395).

Acknowledgments. IP and MVG thank the Mount Stromlo Observatory for its hospitality and financial support. This work is also supported by the Mexican Foundation CONACyT under project No. 36078-E.

\section{References}

Block, D.L., et al. 1994, A\&A, 288, 365

Block, D.L., Puerari, I. 1999, A\&A, 342, 627

Cruz-González, I., et al. 1994, In: Crawford D. L., Craine E.R. (eds.), Instrumentation in Astronomy VIII, Proc. SPIE 2198, 774

Hernández-Toledo, H.M., Puerari, I. 2001, A\&A, 379, 54

Karachentsev, I.D. 1972, Catalogue of isolated pairs of galaxies in northern hemisphere, Soobshch. Spets. Astrofiz. Obs., 7, 3

Puerari, I., Block, D.L., Elmegreen, B.G., Frogel, J.A., Eskridge, P.B. 2000, A\&A, 359, 932

Laurikainen, E., Salo, H. 2000, A\&AS, 141, 103 